\title{
Ordering and Magnetoelastic Properties of Fe-Ga Alloy
}

\author{
K. Perduta ${ }^{a, *}$, J. Olszewski $^{b}$, S. Busbridge $^{a}$ \\ AND M. NABIAEEK ${ }^{b}$ \\ ${ }^{a}$ School of Environment and Technology, University of Brighton \\ Lewes Road, BN2 4GJ Brighton, United Kingdom \\ ${ }^{b}$ Institute of Physics, Czȩstochowa University of Technology \\ al. Armii Krajowej 19, 42-200 Czȩstochowa, Poland
}

In this paper the investigations of the ordering, microstructure and magnetomechanical properties of the $\mathrm{Fe}_{80} \mathrm{Ga}_{20}$ alloy and its composites are presented. The composites consisted of the $\mathrm{Fe}-\mathrm{Ga}$ particles with a size distribution in the range between 20 and $50 \mu \mathrm{m}$. These particles were produced by blade-milling of arc-melted alloy. After milling the powders were annealed at $723 \mathrm{~K}$ for $2.5,7.5$, and $12.5 \mathrm{~h}$. The epoxy-bonded composites in rectangular shape were made from the as-cast as well as from the annealed powders. The Mössbauer spectroscopy studies revealed that the as-cast alloy is completely disordered. After annealing at $723 \mathrm{~K}$ the long range atomic order appeared. It was stated that the best magnetoelastic properties were exhibited by composites made from ferromagnetic particles obtained from the as-cast alloy. The measured saturation magnetostriction was $\lambda_{\mathrm{s}}=70 \times 10^{-6}$ for the bulk alloy and $\lambda_{\mathrm{s}}=100 \times 10^{-6}$ for composite.

PACS numbers: 76.80.+y, 75.50.Bb, 75.80.+q, 81.40.Ef, 83.80.Ab

\section{Introduction}

It has been recognized that substituting approximately 20 at.\% of nonmagnetic $\mathrm{Ga}$ for Fe in bcc solid solution resulted in a tenfold increase in magnetostriction, $\lambda_{\mathrm{s}}$, over that of Fe and twofold increase in $\lambda_{\mathrm{s}}$ in comparison with that for $\mathrm{Fe}-\mathrm{Al}$ alloys $[1,2]$. It is commonly believed that $\mathrm{Ga}-\mathrm{Ga}$ pairing is responsible for this effect in the case of Fe-Ga alloys near 19 at.\% of Ga. The further increase in $\mathrm{Ga}$ amount in $\mathrm{Fe}-\mathrm{Ga}$ alloy $(\approx 25$ at.\%) resulted in creation of an ordered $\mathrm{DO}_{3}$ phase and deterioration of magnetoelastic properties [3].

The single crystals of $\mathrm{Fe}-\mathrm{Ga}$ (up to $\approx 19$ at.\% of Ga) have been shown to exhibit magnetostrictive strains up to $400 \times 10^{-6}$ along the $\langle 100\rangle$ crystallographic direction with relatively low saturating fields $[2,4]$. The $\mathrm{Fe}-\mathrm{Ga}$ alloys have most of the required characteristics of good magnetostrictive transducer materials due

*corresponding author; e-mail: kama@wip.pcz.pl 
to their large saturation magnetostriction at low saturation fields, high mechanical strength, and good ductility. They may be useful in magnetostrictive actuators and sensors, for example linear motors, damping devices, torque sensors, speakers and microphones. These alloys also exhibit good corrosion resistance $[5,6]$.

A significant problem with metallic magnetic actuators is eddy current loss when actuated at high frequencies, which significantly limit their useable bandwidth to typically less than $1 \mathrm{kHz}$. A solution to this problem is to embed the magnetic particles in a non-conducting binder (i.e. create so-called 0-3 composite). Magnetic composites represent a large group of materials consisting of ferromagnetic or superparamagnetic particles dispersed in non-ferromagnetic matrix. The mechanical and magnetic characteristics of these materials depend on the properties of both the particles and the matrix. The smaller particle size substantially reduces eddy currents without a significant lowering of the magnetostriction $[7,8]$. Composites of epoxy-bonded $\mathrm{Fe}-\mathrm{Ga}$ alloys possess additional benefits of easy manufacturability and low material costs.

In this paper we present studies of the microstructure and magnetic properties of bulk and epoxy-bonded Fe-Ga particle composites.

\section{Experimental procedures}

Ingots of the initial $\mathrm{Fe}_{80} \mathrm{Ga}_{20}$ alloy were obtained by arc melting of high purity components. In order to achieve the homogeneity the ingots were remelted several times. Subsequent blade-milling in an argon atmosphere and sieving of powders provided particles in the size range of $20-50 \mu \mathrm{m}$. After blade-milling, the powders were annealed at $723 \mathrm{~K}$ for $2.5,7.5$ and $12.5 \mathrm{~h}$. The various composites were made from powders directly after blade-milling and after each stage of annealing. The powders were mixed with an epoxy binder and compressed under a pressure of $120 \mathrm{MPa}$ to obtain rectangular samples with dimensions of $10 \mathrm{~mm} \times 40 \mathrm{~mm}$ and thickness of 7-10 mm. Compaction was carried out without an applied magnetic field. The volume fraction of the $\mathrm{Fe}-\mathrm{Ga}$ powder used was nominally 0.80 .

The microstructure of the powdered alloy was studied using the Mössbauer spectroscopy. This technique was chosen because X-ray diffraction is relatively insensitive to ordering in $\mathrm{Fe}-\mathrm{Ga}$ alloys (i.e. the atomic scattering factors in this system result in extremely weak superlattice peaks). The Mössbauer studies were carried out for the powders obtained from the as-cast alloy and after annealing at $723 \mathrm{~K}$ for $2.5,7.5$, and $12.5 \mathrm{~h}$. The saturation magnetostriction $\left(\lambda_{\mathrm{s}}\right)$ of the bulk alloy and its composites was measured using a standard strain gauge technique. All investigations were performed at room temperature.

\section{Results and discussion}

It is well known that the $\mathrm{Fe}_{x} \mathrm{Ge}_{1-x}$ alloys exhibit a strong correlation between the structure and their magnetoelastic properties. In order to examine the 
existence of the ordered phase in this alloy, the Mössbauer spectra for $\mathrm{Fe}_{80} \mathrm{Ga}_{20}$ powders were obtained.

All Mössbauer spectra of FeGa powders have the Zeeman sextet shape with broadened lines (Fig. 1). They were decomposed into five independent elementary subspectra corresponding to iron atoms having $n=0,1,2,3$ or 4 gallium atoms in their nearest neighbourhood (NN).

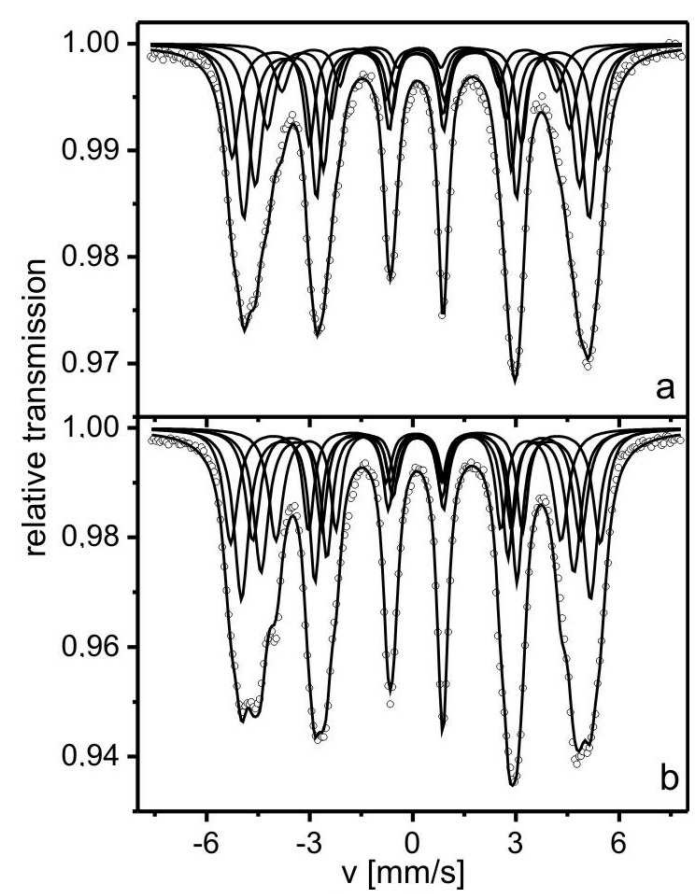

Fig. 1. Transmission Mössbauer spectra and their components of the as-cast (a) and annealed at $723 \mathrm{~K}$ for $7.5 \mathrm{~h}$ (b) powdered $\mathrm{Fe}_{80} \mathrm{Ga}_{20}$ alloy.

The hyperfine interaction parameters obtained from the Mössbauer spectra analysis are shown in Table. It may be added that the values obtained for $\mathrm{Fe}_{80} \mathrm{Ga}_{20}$ powders annealed for $12.5 \mathrm{~h}$ were almost the same as for the samples annealed for $7.5 \mathrm{~h}$ (they are not presented in Table).

Taking into account the values of the hyperfine magnetic field induction and the isomer shift of the elementary sextets (Table) and using the additive model $[9,10]$ they may be approximated by

$$
B_{m}(n)=B_{m}^{\mathrm{Fe}}+n \Delta B^{\mathrm{Ga}}
$$

and

$$
\mathrm{IS}(n)=\mathrm{IS}_{0}+n \Delta \mathrm{IS}^{\mathrm{Ga}},
$$

where $B_{m}^{\mathrm{Fe}}$ is the hyperfine magnetic field induction of pure iron, $n$ is the number of $\mathrm{Ga}$ atoms in the first coordinating zone of an Fe atom, $\Delta B^{\mathrm{Ga}}$ is the change of 
TABLE

Hyperfine interaction parameters for elementary Zeeman sextets for the as-cast and annealed $\mathrm{Fe}_{80} \mathrm{Ga}_{20}$ powder; $B$ - hyperfine magnetic field induction, IS - isomer shift (relative to $\alpha$-Fe) and Int - relative intensity of elementary sextet.

\begin{tabular}{|c|c|c|c|c|c|}
\hline \multirow{2}{*}{$\begin{array}{c}\text { Number } \\
\text { of Ga } \\
\text { atoms }\end{array}$} & \multicolumn{2}{|c|}{ Mössbauer parameters } & \multicolumn{3}{|c|}{ Heat treatment } \\
\hline & & & as-cast & $723 \mathrm{~K} / 2.5 \mathrm{~h}$ & $723 \mathrm{~K} / 7.5 \mathrm{~h}$ \\
\hline \multirow{3}{*}{$n=0$} & I sextet & $B[\mathrm{~T}]$ & 33.04 & 33.22 & 33.29 \\
\hline & & IS $[\mathrm{mm} / \mathrm{s}]$ & 0.077 & 0.081 & 0.080 \\
\hline & & Int [\%] & 19.0 & 20.1 & 17.6 \\
\hline \multirow[t]{3}{*}{$n=1$} & II sextet & $B[\mathrm{~T}]$ & 31.16 & 31.37 & 31.40 \\
\hline & & IS $[\mathrm{mm} / \mathrm{s}]$ & 0.106 & 0.098 & 0.099 \\
\hline & & Int [\%] & 32.1 & 24.9 & 25.2 \\
\hline \multirow[t]{3}{*}{$n=2$} & III sextet & $B[\mathrm{~T}]$ & 29.24 & 29.46 & 29.43 \\
\hline & & IS $[\mathrm{mm} / \mathrm{s}]$ & 0.134 & 0.121 & 0.120 \\
\hline & & Int [\%] & 27.2 & 17.0 & 16.0 \\
\hline \multirow[t]{3}{*}{$n=3$} & IV sextet & $B[\mathrm{~T}]$ & 27.24 & 28.19 & 28.15 \\
\hline & & $\mathrm{IS}[\mathrm{mm} / \mathrm{s}]$ & 0.162 & 0.141 & 0.139 \\
\hline & & Int [\%] & 15.0 & 19.9 & 21.9 \\
\hline \multirow[t]{3}{*}{$n=4$} & V sextet & $B[\mathrm{~T}]$ & 25.01 & 25.67 & 25.61 \\
\hline & & IS $[\mathrm{mm} / \mathrm{s}]$ & 0.186 & 0.159 & 0.157 \\
\hline & & Int $[\%]$ & 6.7 & 18.1 & 19.3 \\
\hline
\end{tabular}

Quadrupole splitting for all sextets is equal to zero.

the hyperfine magnetic field induction of $B_{m}^{\mathrm{Fe}}$ caused by the presence of one Ga atom, $\mathrm{IS}_{0}$ is a constant and $\Delta \mathrm{IS}{ }^{\mathrm{Ga}}$ is the change of the isomer shift caused by the presence of one Ga atom in the $\mathrm{NN}$ of an Fe atom.

It has been found that for the as-cast powders $B_{m}^{\mathrm{Fe}}=(33.2 \pm 0.2) \mathrm{T}$ and $\Delta B^{\mathrm{Ga}}=(-2.00 \pm 0.07) \mathrm{T}$. However, for the annealed powders $B_{m}^{\mathrm{Fe}}=(33.2 \pm 0.2) \mathrm{T}$ and $\Delta B^{\mathrm{Ga}}=(-1.85 \pm 0.09) \mathrm{T}$.

In the case of the isomer shift for the as-cast powders $\mathrm{IS}_{0}=$ $(0.077 \pm 0.007) \mathrm{mm} / \mathrm{s}, \Delta \mathrm{IS}^{\mathrm{Ga}}=(0.028 \pm 0.005) \mathrm{mm} / \mathrm{s}$ and for the annealed powders $\mathrm{IS}_{0}=(0.081 \pm 0.007) \mathrm{mm} / \mathrm{s}$ and $\Delta \mathrm{IS}^{\mathrm{Ga}}=(0.019 \pm 0.006) \mathrm{mm} / \mathrm{s}$.

Comparing theoretical predictions of the relative intensities $\operatorname{Int}(n)$ of elementary Zeeman sextets with these experimentally achieved, the type of superstructure and its order parameter can be determined. In the case of the $\mathrm{DO}_{3}$ superstructure $\operatorname{Int}(n)$ depends on the probability $(\gamma)$ of finding a Ga atom in the $\mathrm{NN}$ zone of an Fe atom in this type of superstructure [11] as follows:

$$
\operatorname{Int}(n, \gamma)=\frac{0.75-c(1-\gamma)}{1-c}\left\{\frac{1}{3} C_{8}^{n}\left[\frac{c(1-\gamma)}{0.75}\right]^{n}\left[1-\frac{c(1-\gamma)}{0.75}\right]^{8-n}\right.
$$




$$
\begin{aligned}
& +\frac{2}{3} \sum_{j=0}^{n} C_{4}^{j}\left(\frac{c \gamma}{0.25}\right)^{j}\left(1-\frac{c \gamma}{0.25}\right)^{4-j} C_{4}^{n-j} \\
& \left.\times\left[\frac{c(1-\gamma)}{0.75}\right]^{n-j}\left[1-\frac{c(1-\gamma)}{0.75}\right]^{4-n+j}\right\} \\
& +\frac{0.25-c \gamma}{1-c} C_{8}^{n}\left[\frac{c(1-\gamma)}{0.75}\right]^{n}\left[1-\frac{c(1-\gamma)}{0.75}\right]^{8-n},
\end{aligned}
$$

where $c$ is concentration of Ga atoms in the alloy.

The Bragg-Williams long range order parameter $(S)$ [12] can be calculated as

$$
S=\frac{\gamma-c}{0.75}
$$

while for short range ordered structure

$$
\operatorname{Int}\left(n, \alpha_{1}\right)=C_{n}^{8}\left[c\left(1-\alpha_{1}\right)\right]^{n}\left[1-c\left(1-\alpha_{1}\right)\right]^{8-n},
$$

where $\alpha_{1}$ is the Cowley-Warren short range order parameter for first coordination zone $[10,13]$.

In the case when mixture of ordering occurs the relative intensities $\operatorname{Int}(n)$ of the elementary Zeeman sextets can be described as

$$
\left.\operatorname{Int}(n)=V(\operatorname{Int}(n, \gamma))+(1-V) \operatorname{Int}\left(n, \alpha_{1}\right)\right),
$$

where $V$ is the volume fraction of the sample occupied by the $\mathrm{DO}_{3}$-type ordered phase.

From the analysis of the relative intensities of the elementary sextets (Table) it can be seen that in the as-cast powdered samples the atomic ordering does not occur. However, for the annealed powders the $\mathrm{DO}_{3}$-type superstructure $(V=0.5)$ with the Bragg-Williams long range order parameter $S=0.8$ and antiphase boundaries are observed. It is worth adding that in these boundaries short range atomic order is present. The results from this analysis show that in the first coordinating zone of an Fe atom a larger number of Ga atoms are present than is expected from consideration of the Ga concentration in the sample. The Cowley-Warren short range order parameter of this type of antiphase boundaries is $\alpha_{1}=-0.2$. After annealing the powders for 7.5 and $12.5 \mathrm{~h}$ only an increase in the short range order parameter is observed $\left(\alpha_{1}=-0.4\right)$.

The investigations indicate that the best magnetoelastic properties are obtained for the composite made from the as-cast powder. The saturation magnetostriction observed for this composite is equal to $\lambda_{\mathrm{s}}=100 \times 10^{-6}$ (Fig. 2), while $\lambda_{\mathrm{s}}=70 \times 10^{-6}$ for the bulk alloy. When the magnetic field decreases the magnetostriction of composites samples exhibits magnetostriction hysteresis. The hysteresis may be associated with a mechanical interaction between the epoxy matrix and the metallic $\mathrm{Fe}-\mathrm{Ga}$ particles. 


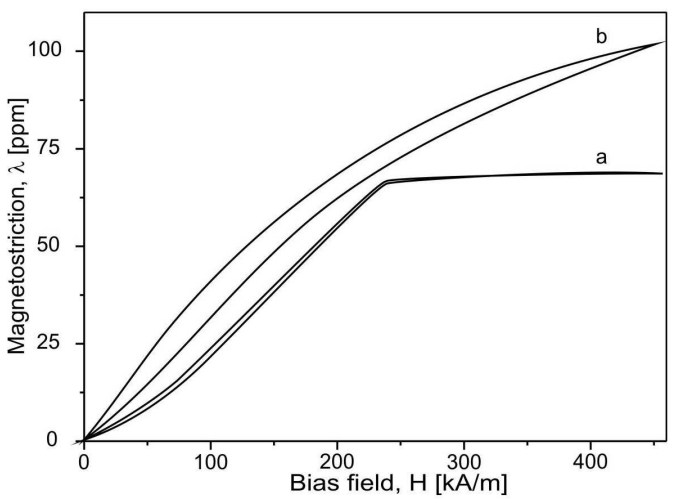

Fig. 2. Magnetostriction curves for the bulk sample of $\mathrm{Fe}_{80} \mathrm{Ga}_{20}(a)$ and its composite made from the as-cast powder with particle size range of $20-50 \mu \mathrm{m}(b)$.

The processes of creep and relaxation of the epoxy matrix may influence the observed magnetostriction hysteresis. A change of the sample size is accompanied by the elongation of the walls of the epoxy-matrix existing between the particles in composite. As the matrix walls become thinner the stress to which they are subjected increases. An additional elongation of the sample occurs when it is placed in a magnetic field. It may be supposed that during decrease in the magnetic field the elongated matrix walls exhibit a tendency to relax stress, which is observed as creep effect. This effect will be more pronounced at larger volume fractions.

\section{Conclusion}

From the Mössbauer spectroscopy studies we have concluded that $\mathrm{Fe}_{80} \mathrm{Ga}_{20}$ alloy obtained by blade-milling of arc melted ingots does not exhibit atomic order. However, after annealing of the powders a mixture of $\mathrm{DO}_{3}$-type superstructure and a short range ordered phase occur. Moreover, we have found that best magnetoelastic properties are exhibited by composites made from ferromagnetic powders obtained from the as-cast alloy. The measured saturation magnetostriction was $\lambda_{\mathrm{s}}=70 \times 10^{-6}$ for the bulk alloy and $\lambda_{\mathrm{s}}=100 \times 10^{-6}$ for composites fabricated from these powders.

\section{Acknowledgments}

This work acknowledges support from the European Union (Interreg IIIa grant number 349).

\section{References}

[1] J.R. Cullen, A.E. Clark, M. Wun-Fogle, J.B. Restorff, T.A. Lograsso, J. Magn. Magn. Mater. 226-230, 948 (2001).

[2] A.E. Clark, M. Wun-Fogle, J. Restorff, T.A. Lograsso, A.R. Ross, D.L. Schlagel, IEEE Trans. Magn. 36, 3238 (2000). 
[3] T.A. Lograsso, A.R. Ross, D.L. Schlagel, A.E. Clark, W. Wun-Fogle, J. Alloys Comp. 350, 95 (2003).

[4] R. Kellogg, A.B. Flatau, A.E. Clark, M. Wun-Fogle, T.A. Lograsso, J. Appl. Phys. 91, 7821 (2002).

[5] N. Srisukhumbowornchai, S. Guruswamy, J. Appl. Phys. 90, 5680 (2001).

[6] T.V. Jayaraman, N. Srisukhumbowornchai, S. Guruswamy, M.L. Free, Corrosion Sci. 49, 4015 (2007).

[7] S. Bednarek, Appl. Phys. A. 68, 63 (1999).

[8] A. Clark, U.S. Patent No. 4,378,258, March 29 (1983).

[9] G.K. Wertheim, V. Jaccarino, J.H. Wernick, D.N.E. Buchanan, Phys. Rev. Lett. 12, 24 (1964).

[10] S.M. Dubiel, W. Zinn, J. Magn. Magn. Mater. 45, 298 (1984).

[11] V.S. Litvinov, S.D. Karakishev, V.V. Ovchinnikov, Nuclear gamma-resonance spectroscopy of alloys, Metallurgiya, Moskva 1982, p. 58 (in Russian).

[12] A.A. Smirnov, Generalized ordering theory of alloys, Naukova Dumka, Kiev 1986, p. 71 (in Russian).

[13] M.A. Krivoglaz, A.A. Smirnov, Theory of ordering in alloys, Fizmatizd, Moskva 1958, p. 68 (in Russian). 